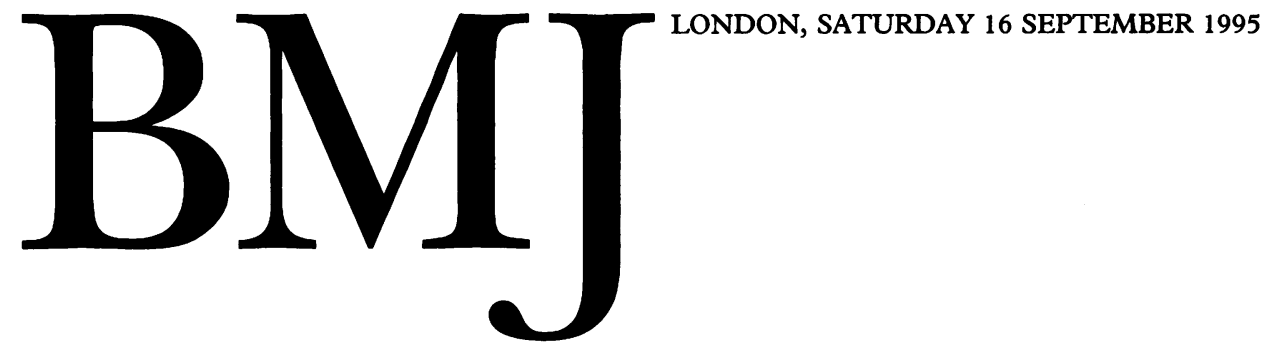

\title{
Breast cancer and hormonal supplements in postmenopausal women
}

\author{
Among current users of combined supplements the risk rises with five or more years' treatment
}

Breast cancer is a hormonal cancer that is common after the menopause, and hence the effects of hormone supplementation are of enormous concern to women. The epidemiology of breast cancer remains largely unexplained, as known risk factors explain only around $40 \%$ of the disease. This is due to its prolonged, complicated, and multifactorial aetiology. Studies suffer from subtle selection processes that lead to unmeasured confounding, from long delays in measuring the effects on early carcinogenic changes, and from imprecision in investigations of modifying effects among important subgroups.

Hormone supplements are clearly effective in relieving menopausal symptoms and preventing osteoporosis if taken for long enough. Do these and the putative protective effect on coronary heart disease ${ }^{1}$ make them the most important advance in preventive medicine in the past 50 years? ${ }^{2}$ Endometrial cancer is a serious problem with unopposed oestrogen, and, assuming that the protection from heart disease is real, breast cancer might still be a serious problem with either oestrogen or combined (opposed) preparations. How might these considerations balance out for a woman wanting to understand the benefits and risks? For a 50 year old woman the baseline lifetime risk of coronary heart disease is around $45 \%$, of hip fracture is $15 \%$, and of breast cancer is $8 \%$. Clearly, such aggregate data will weigh differentially with the potential relief of symptoms, prevention of fractures, and side effects in each woman as well as her family history, risk factors, and fears and preferences.

Most of the epidemiological studies with a long follow up measure the effect of oestrogen alone, which was used before combined supplements were introduced in the 1980 s because of the risks of inducing endometrial cancer with unopposed oestrogen. Meta-analyses have shown that long term use (more than 10 years in total) may increase the risk of breast cancer by $50 \%$ but that shorter durations of use do not affect the risk. ${ }^{34}$

Pike et al had predicted that the addition of progestin would increase the risk of breast cancer ${ }^{5}$ as most proliferative activity occurs in the luteal phase of a cycle, when both oestrogen and progesterone are produced. Gambrell and colleagues' study led people to hope that combined supplements might reduce the risk, ${ }^{6}$ but the authors failed to adjust for known confounding and to account for possible long delays in any increased risk in breast cancer diagnosis. On the basis of study of only 10 patients who developed breast cancer Bergkvist more recently indicated a worrying increase in risk, with a relative risk of 4.4 for six or more years (but with wide confidence intervals). ${ }^{7}$

An updated analysis of the data from the American nurses' health study was published recently. ${ }^{8}$ This study reports the risk of a diagnosis of breast cancer and death according to use of hormone supplements, with considerable information on women using combined supplements-some $20 \%$ of the total exposure to supplements in their study of 120000 women. These results show that combined supplements do not reduce the incidence of or mortality from breast cancer. In fact among current users the risk rises with five or more years' use.

Although more older women die of heart disease than breast cancer, the effects of hormonal supplements on total mortality may nevertheless cancel out, particularly around the menopause. Risk benefit studies comparing net years of life gained from the age of 50 indicate that the true effect of hormone supplements on breast cancer will be the crucial determinant of the ultimate balance of risks and benefits. ${ }^{9}$ If the effects of long term use are moderate and late a net average gain in lifespan is most likely if supplements are taken for 10 years from the age of 50 . For longer use such net benefits might then improve if a longer effect on coronary heart disease and no prolonged effect on breast cancer are assumed.

\section{Duration of adverse effects unknown}

The nurses' health study indicates that current use of any hormonal supplement for more than five years will increase the risk of breast cancer by around $50 \%$. The effect is stronger for older women, with a $70 \%$ increase for women aged 60 to 64. If the effect is associated only with current use or is prolonged after supplements are stopped the choices will be different. In this study the effect lasted for two years after the women stopped using the supplements, but, as the authors state, "breast cancer cases among women taking estrogen plus progestin precluded detailed analysis of the risk according to duration of use and age." 8 Thus reliable information on the effect of the duration of use of combined supplements and on how long these risks might continue after long term use is still lacking.

The great enthusiasm for prophylactic use of menopausal hormones has relied on hopes that combined supplements would reduce the risk of breast cancer, which would itself have a lower mortality. Both these are now less plausible. These new estimates of risk for breast cancer will probably, however, still give rise to a net gain in years of life so long as 
the effects on risk revert to normal after the supplements are stopped and the protective effects on heart disease last long after they are stopped. If the effects on breast cancer last rather longer than expected after, say, 10 years' use then hormone supplements could be responsible for an average net loss of years of life. If, secondly, the attributable effects of combined supplements on deaths from heart disease are rather less than currently expected after the supplements are stopped then longer use may also result in an aggregate net loss. Neither of these hypotheses is implausible.

The balance with prevention of osteoporosis will then be further compromised, ${ }^{10}$ and cost effectiveness will depend crucially on improved quality of life $^{11}$ owing to relief of symptoms and prevention of fractures, to be set against a possible net loss of years of life. For women with a higher baseline risk of breast cancer the symptom thresholds will then be more severe still. With the falling baseline risk of heart disease, the balance will move away from the prophylactic use of supplements, whether or not these two hypotheses turn out to be correct.

The present data suggest that users who have been on supplements for more than five years are at increased risk of breast cancer. The net effect on years of life might still be positive as longer term use may be much less attractive for precisely that reason; women are concerned by such risks and might be inclined to stop before five years. ${ }^{12}$
I would like to thank Edel Daly, of the department of public health and primary care, Oxford, for analytical help.

KLIM MCPHERSON Professor of public health epidemiology

Health Promotion Sciences Unit,

Department of Public Health and Policy,

London School of Hygiene and Tropical Medicine,

London WC1E 7HT

1 Petitti DB. Coronary heart disease and estrogen replacement therapy. Can compliance bias explain the results of observational studies? Ann Epidemiol 1994;4:115-8.

2 Studd J. Complications of hormone replacement therapy in postmenopausal women. $f \mathrm{R}$ Soc $\mathrm{Med}$ 1992;85:376-8.

3 Grady D, Rubin SM, Petitii DB, Fox CS, Black D, Ettinger B, et al. Hormone therapy to prevent disease and prolong life in postmenopausal women. Ann Intern Med 1992;117:1016-37.

4 Colditz GA, Egan KM, Stampfer MJ. Hormone replacement therapy and risk of breast cancer: Results from epidemiologic studies. Am $\mathcal{O}$ Obstet Gynecol 1993;186:1473-80.

5 Pike MC, Spicer DV, Dahmoush I Press MF. Estrogens, progestogens, normal breast cell proliferation, and breast cancer risk, Epidemiol Rev 1993;15:17-35.

proliferation, and breast cancer risk. Epidemiol Rev 1993;15:17-35.
Gambrell RD Jr, Maier RC, Sanders BI. Decreased incidence of breast cancer in postmenopausal estrogen-progestogen users. Obstet Gynecol 1983;62:435-43.
entrell RD Jr, Maier RC, Sanders BI. Decreased incidence

7 Bergkvist L, Adami H-O, Persson I, Hoover R, Schairer C. The risk of breast cancer after estrogen and estrogen-progestin replacement. N Engl f Med 1989;321:293-7.

8 Colditz GA, Hankinson SE, Hunter DJ, Willett WC, Manson JE, Stampfer MJ, et al. The use of estrogens and progestins and the risk of breast cancer in postmenopausal women. $N$ Engl $f \mathrm{Med}$ 1995;332:1589-93.

9 Daly E, Vessey P, Barlow D, Gray A, McPherson K, Roche M. Hormone replacement therapy in a risk-benefit perspective. In: Berg G, Hammar M, eds. The modern management of the menopause; a perspective for the 21 st century. The proceedings of the VII international congress on the menopause, Stockholm, Sweden 1993. Parthenon, 1994:473-97.

10 Felson DT, Zhang Y, Hannan MT, Kiel DP, Wilson PWF, Anderson JJ. The effect of postmenopausal estrogen therapy on bone density in elderly women. $N$ Engl $f$ Med 1993:329:1141-6.

11 Daly E, Gray A, Barlow D, McPherson K, Roche M, Vessey M. Measuring the impact of menopausal symptoms on quality of life. $B M 7 f 1994 ; 307: 836-40$

12 Randall S. Problems encountered by long-term hormone replacement therapy users. British fournal of Family Planning 1990;16:101-5.

\section{After a first episode of venous thromboembolism}

\section{Stop anticoagulant treatment after four to six weeks in patients with "reversible" risk factors}

Several advances in the management of venous thromboembolism with anticoagulants have occurred over the past few years. The importance of rapid and adequate treatment with heparin is well established. So is the early introduction of oral warfarin, ${ }^{1}$ which results in patients being given heparin for shorter periods and having shorter stays in hospital compared with days gone by. Nomograms for warfarin have made initial dosing easier and more predictable. ${ }^{2}$ The widespread use of the international normalised ratio for reporting the effect of warfarin has resulted in more universally accepted recommendations regarding the desirable degree of anticoagulation in given clinical settings.

Two aspects of management require further systematic investigation: the correct place to be assigned to low molecular weight heparins and (the topic to be discussed here) the optimal duration of oral anticoagulant treatment after a first symptomatic thromboembolic event.

Despite pages covering mechanisms, drug interactions, and other information, instructions regarding the optimal duration of treatment in most textbooks are refreshingly terse and to the point: "Anticoagulation with warfarin is usually continued for 3-6 months." Full stop. But who should receive oral anticoagulants for three months? For four and a half months? For six months? Why? Should some people receive them for longer and some for shorter periods? Can we do better armed with published data, which have been reviewed recently by Hirsh? ${ }^{3}$

The natural course of venous thromboembolism is one of recurrence, more commonly in the ipsilateral leg or with pulmonary embolism. Without treatment, the risk of recurrence is of the order of $22 \%$ in the first four weeks after discharge from hospital, although the risk falls rapidly in subsequent weeks. ${ }^{4}$ Treatment with oral anticoagulants reduces the incidence of recurrent venous thromboembolism from about $25 \%$ within three months after discharge to less than $4 \% .^{3}$ Nevertheless, as reported in a prospective cohort study of 355 patients ${ }^{5}$ and in a recent Swedish randomised study designed to determine the optimal duration of oral anticoagulant treatment, ${ }^{6}$ attrition continues after oral anticoagulants are suspended. In the cohort study, at six months, two years, five years, and eight years after the initial event there was a cumulative incidence of recurrence of $8.6 \%$, $17 \cdot 5 \%, 24 \cdot 6 \%$, and $30 \cdot 3 \%$ respectively. ${ }^{5}$ In the second of these the rate of recurrence of venous thromboembolism after oral anticoagulants were stopped was identical between six months after discharge and the two year follow up irrespective of whether the initial treatment was for six weeks or six months. ${ }^{6}$

In a study by the research committee of the British Thoracic Society 712 patients with venous thromboembolism were randomised to receive anticoagulants for either four weeks or 12 weeks after the start of treatment with heparin. ${ }^{7}$ The patients were followed up for 12 months. The recurrence rate was higher $(7 \cdot 8 \%)$ in those patients given anticoagulants for four weeks as opposed to 12 weeks (4\%). Of the total of 116 patients in both treatment groups who had postoperative venous thromboembolism, however, only one had a recurrent event. Thus, regardless of the duration of oral anticoagulant treatment the risk was minimal. In the 596 medical patients the overall recurrence rate was $6.9 \%$, with a significant preponderance $(9.1 \%)$ in those treated for four weeks compared with those treated for 12 weeks $(4 \cdot 7 \%)$.

In the recent Swedish trial 897 patients were randomly assigned to receive oral anticoagulants for six weeks or six months after at least five days' treatment with heparin. ${ }^{6}$ After two years' follow up recurrence was significantly more likely 